\title{
The Usage of the Johnson-Cook Constitutive Model in the Finite Element Analysis of the Caulking Process
}

\author{
Claudiu Rodean ${ }^{1, *}$, Livia-Dana Beju $^{2}$, Gabriela Rusu$^{2}$, and Mihai Popp ${ }^{2}$ \\ ${ }^{1}$ Continental Automotive Systems SRL, 8 Salzburg Street, Sibiu, Romania \\ 2"'Lucian Blaga" University of Sibiu, Faculty of Engineering, 4 Emil Cioran Street, Sibiu, Romania
}

\begin{abstract}
The present paper highlights the importance of generating a model for analysing the caulking process. The caulking operation is a fast, cost-efficient, cost-effective way of assembling, which is currently less studied in world-wide papers. The operation is at the border between plastic deformation and cutting process. It is therefore necessary to create a model to describe the process. In the paper are presented the main examples of applications, the main parameters that characterize the process, the current state of the cutting and plastic deformation models. After the analysis we concluded that the most valuable model for describing plastic deformations is the Johnson-Cook model. This information is the basis for developing a model for describing the caulking process.
\end{abstract}

\section{Introduction}

A consequence of the evolution of today's industries is the emergence of competitiveness between companies, the need to invest more resources in the research of different fields. In the automotive, aeronautical, medical or energetic industries, there are needed reliable, quick and cost-effective assemblies.

One of the most modern solutions is the caulking process, which, using a tool as used in the cutting operations (with edges having specific cutting angles) performs cutting and deformation of a material volume that will be pressed. As a result, the non-destructive assembly of the two components will be obtained, as it can be seen in the following picture (Fig 1).

\footnotetext{
* Corresponding author: claudiu rod90@yahoo.com
} 


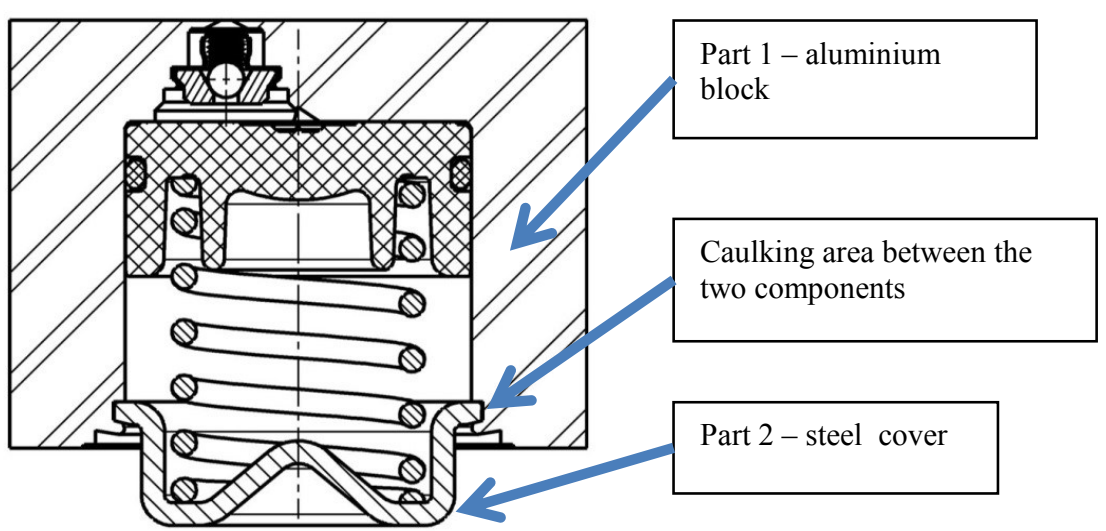

Fig. 1. Presentation of the caulking area between one part of aluminium (the block) and one part of steel (the cover).

Products such as ABS braking systems (as it can be seen in figure 2) and shock absorbers (visible in figure 3) contain subassemblies that are obtained by the caulking process.

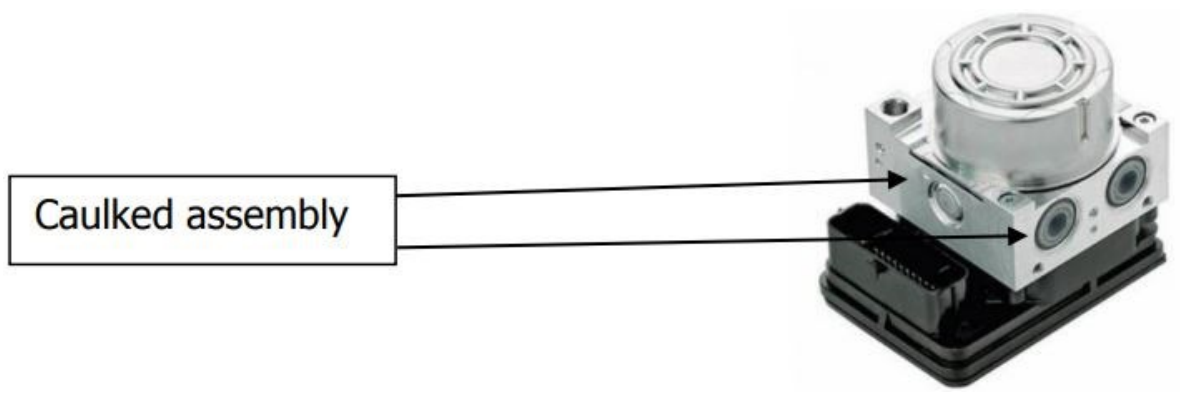

Fig. 2. Caulked assembly used at brake systems.

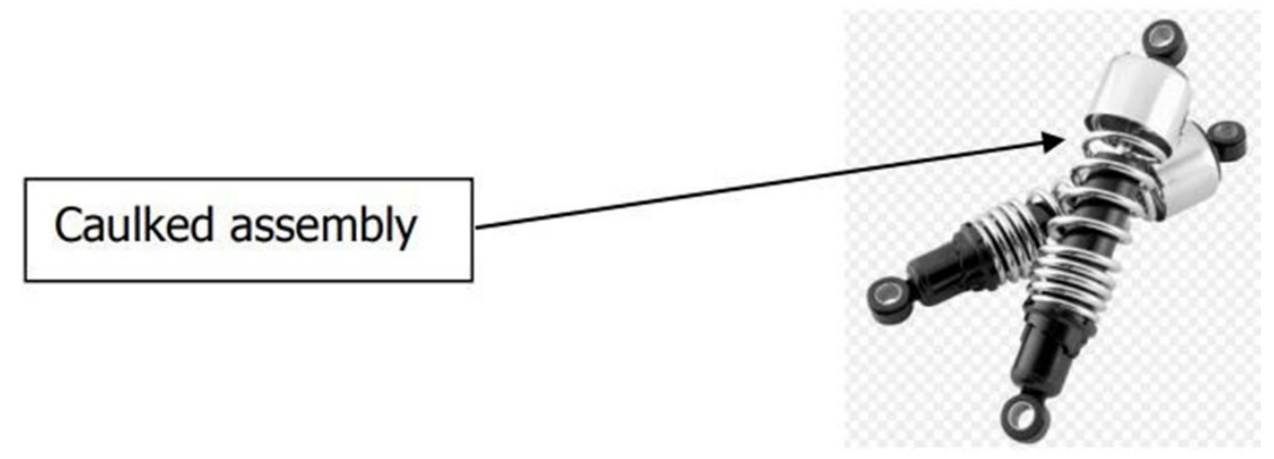

Fig. 3. Caulked assembly used at shock absorbers systems.

The caulked assembly has to fulfil the following functions:

- Sealing to liquid and gas transfer over a temperature range set by the recipient, generally between $-40^{\circ} \mathrm{C}$ to $120^{\circ} \mathrm{C}$,

- Resistance to high internal pressure,

- A continuous caulked area. 


\section{Parameters that characterize the caulking process}

Figure 4 shows the situation of a steel cover that is assembled on a high plasticity part (generally aluminium alloys). Notice the position of the cutting tool that will cut and then deform the work piece material. Particular attention must be paid to the shape of the rank surface which plays a decisive role in the subsequent deformation of the material.

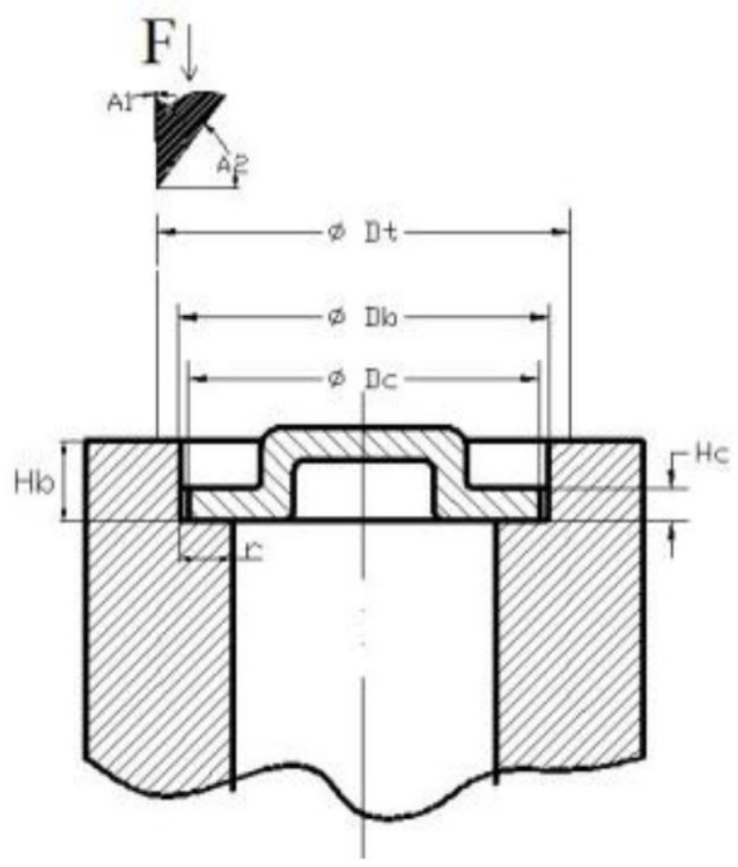

Fig. 4. Geometrical parameters of the caulking process (before deformation).

The geometrical parameters of the cover and the body which are directly related to the caulking process are the following ones:

- $\mathrm{Hb}$ - the height of the material (mostly related to the quantity of material) which is cut and pressed by the tool;

- $\mathrm{Hc}$ - the height of the cover;

- $\varnothing \mathrm{Db}$ - the diameter of the body, (also related to the quantity of the material);

- $\quad \varnothing D c-$ the diameter of the cover;

- $\quad \varnothing \mathrm{Dt}$ - the diameter of the caulking tool;

- A1 - clearance angle of the caulking tool.

- A2 - rank angle of the caulking tool.

Together with the geometric parameters, the caulking process demands also a list of technological parameters:

- $\mathrm{t}$ - Tool pressing time.

- F - Caulking force.

- The accuracy of the devices: perpendicularity; linearity etc.

After the caulking process is performed, there are several output parameters which occurs. The geometrical parameters can be seen in the following picture (Fig 5): 


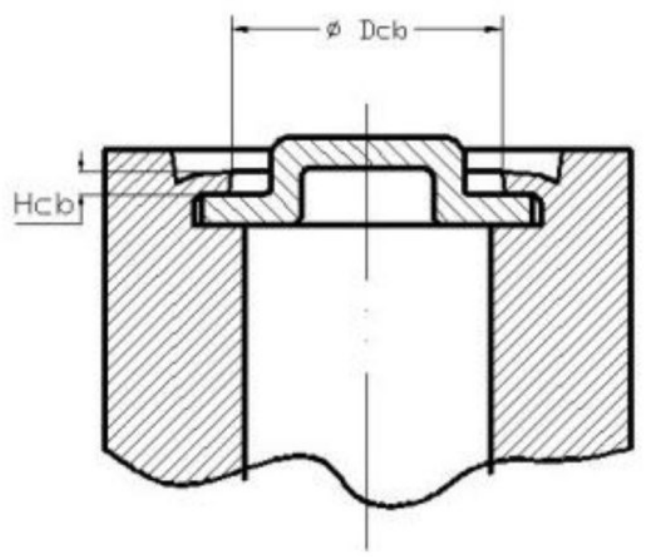

Fig. 5. Geometrical parameters after the caulking process.

The output geometrical parameters are:

- $\varnothing \mathrm{Dcb}$ - the actual diameter of the caulked material.

- $\mathrm{Hcb}$ - the height of the caulked material which is pressed over the cover;

These are the geometric parameters before and after the caulking process. In order to evaluate the assembly, studies are needed on the material deformation, extrusion, nature of material fibres, grit and micro- hardens. It is also necessary to analyse the micro cracks that can be generated in the caulking process and to determine their appearance causes (different materials characteristics, strengths, pressing duration, tool wear, etc.).

\section{State of the art}

A modern way to optimize the product development cycle is the use of finite element analysis that saves both time and money savings. The finite element method is a numerical method used to solve partial derivative equations that model physical systems with an infinite number of degrees of freedom. The finite element method is a generalization of the classical variational methods (Rayleigh-Ritz) and the weighted residue (Galerkin), the smallest squares, the colocation, etc. The fundamental idea of the finite element method is that the given area of the problem is represented as an ensemble of sub-regions called finite elements. These elements are connected to each other through points known as nodes. On the finite element domain, it is possible to systematically generate approximation functions necessary to solve differential equations describing behaviour by any of the varied or weighted residue methods. [1]

Some of the most important finite element analysis programs include: Abaqus, Ansys, Nastran, Cosmos, Algor, etc. Compatibility between computer assisted design and finite element analysis has long been expected. Models designed by designers are highly complex. Differences between CAD files and FEA programs have long limited the ability of companies to use the CAD-FEA combination. The design resulted in IGES or STEP standard files, which however generated problems in geometry exchange. Newer integrated design environments have been created to eliminate incompatibilities and have broadened the range of files that can be imported by specialized FEA programs, so no longer need to convert files. Abaqus is used in the automotive, aerospace, and industrial industry industries. The product is widely used in academic institutions and research institutions due to materials modelling capabilities. Figure 6 presents the steps of an analysis by Abaqus. 


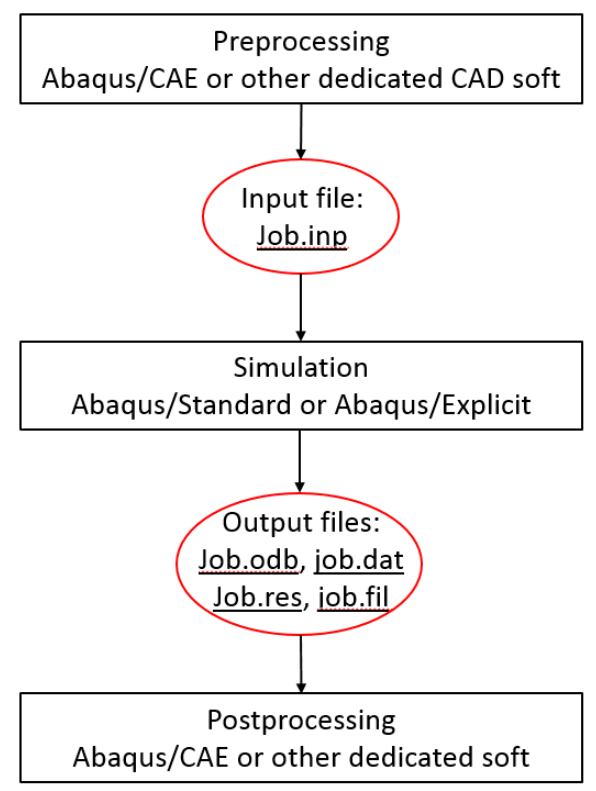

Fig. 6. Stages of a FEM analysis using Abaqus.

The deformability of metals expresses their ability to deform plastic to take a given shape without defects in the piece [1]. Defects that may occur in the plastic deformation of the sheet metal parts differ according to the process of obtaining the part. Thus, when stamping, deep or stretching, various defects may occur, such as: the curl wall or flange; tears at the bottom of the piece, flange or corners; festoons; wrinkle of the side wall; ridges; non coaxialities etc. The factors limiting the deformation of the material by the stamping processes are: cracking, tearing, creasing, and changing the roughness or appearance of the surface of the piece. [2-4]. The factors on which the deformability of the metal sheets depends are the following, according to [1]:

- $\quad$ properties of the material: mechanical, metallurgical, chemical;

- deformation conditions: stress state, speed, temperature of part shape, lubrication;

- criteria for limiting deformation: breaking, looping, corrugation and arcing.

The plastic deformation phenomena have been extensively studied by a large number of researchers who have developed different models that can describe the cutting and deformation operations by clamping.

Models can be categorized into three major categories:

- Empirical constitutive models;

- Semi-empirical constitutive models;

- Physically based constitutive models;

The first two ones, empirical constitutive models and semi-empirical models, such as Cowper-Symonds (CS) model and Johnson-Cook (JC) model, involve less material constants and has been embedded in many finite element codes, thus widely used to predict the mechanical properties of materials. The physically based constitutive model can manifest the deformation behaviour of the material over a wide range of strain rates and temperatures. The big disadvantage of this model is the fact that a large number of experimental data are needed for developing.

The Johnson-Cook constitutive model was released in the year 1983 and it is represented by a phenomenological model (which means it is not based on traditional 
plasticity theory) and reproduces several material responses which can be studied in the processes of impact and penetration of the metals [1]. For this, the three significant material responses are the following:

- Strain hardening;

- Strain-rate effects;

- Thermal softening.

The Johnson-Cook plasticity model is particularly suited to model high-strain-rate deformation of metals. This model is particular type of Mises plasticity that includes analytical forms of the hardening law and rate dependence. It is generally used in adiabatic transient dynamic analysis.

In Abaqus a perfectly plastic material (with no hardening) can be defined, or work hardening can be specified. Isotropic hardening, including Johnson-Cook hardening, is available in both Abaqus/Standard and Abaqus/Explicit. In addition, Abaqus provides kinematic hardening for materials subjected to cyclic loading. Johnson-Cook hardening is a particular type of isotropic hardening where the static yield stress, $\sigma^{0}$, is assumed to be of the form

$$
\sigma^{0}=\left[A+B\left(\varepsilon^{-p l}\right)^{n}\right]\left(1-\hat{\theta}^{m}\right)
$$

where $\varepsilon^{-p l}$ is the plastic equivalent plastic strain and $\mathrm{A}, \mathrm{B}, \mathrm{n}$ and $\mathrm{m}$ are material parameters measured at or below the transition temperature, $\theta_{\text {transition, }} \hat{\theta}$ is the nondimensional temperature defined as

$$
\hat{\theta}=\left\{\begin{array}{cc}
0 & \text { for } \theta<\theta_{\text {transition }} \\
\left(\theta-\theta_{\text {transition }}\right) /\left(\theta_{\text {melt }}-\theta_{\text {transition }}\right) \text { for } \theta_{\text {transition }} \leq \theta \leq \theta_{\text {melt }} \\
1 & \text { for } \theta>\theta_{\text {melt }}
\end{array}\right.
$$

where $\theta$ is the current temperature, $\theta_{\text {melt }}$ is the melting temperature, and $\theta_{\text {transition }}$ is the transition temperature defined as the one at or below which there is no temperature dependence of the yield stress. The material parameters must be measured at or below the transition temperature.

The Johnson-Cook plasticity can be used in conjunction with the Johnson-Cook dynamic failure model in Abaqus/Explicit or with progressive damage and failure models.

The reasons for using a Johnson Cook model are:

- Under dynamic impact, the materials experience effects such as: high strain rates, large strain and elevated temperature;

- The behaviour differs significantly under impact loading, putting material to quasistatic loading approach;

- Researchers have proposed several material model case by case;

- There is no universal model for a large variety of materials, which can account all above parameters for impact simulations;

- Reliable prediction in response to impact loading is critical for accurate design;

- Johnson Cook is a popular constitutive material model for metals, widely used in simulation of impact and penetration problems.

The Johnson-Cook constitutive model has been used by various researchers with the aim of modeling of the cutting processes and also of some plastic deformations. In the following, we will present the results of some studies that can support us in creating a model for caulking. Examples of domains where the Johnson Cook model is used are: automotive applications, high speed machinery, defence applications such as battle tanks, high speed project impact on armour. 
In his paper, Daoud [2] investigates the effect of five different sets of JC constants on the numerical FEM predicted cutting parameters. After studying the predicted results, the author did a comparison between the theoretical results and the ones obtained from the experimental study. The differences can be seen in figure 7 .
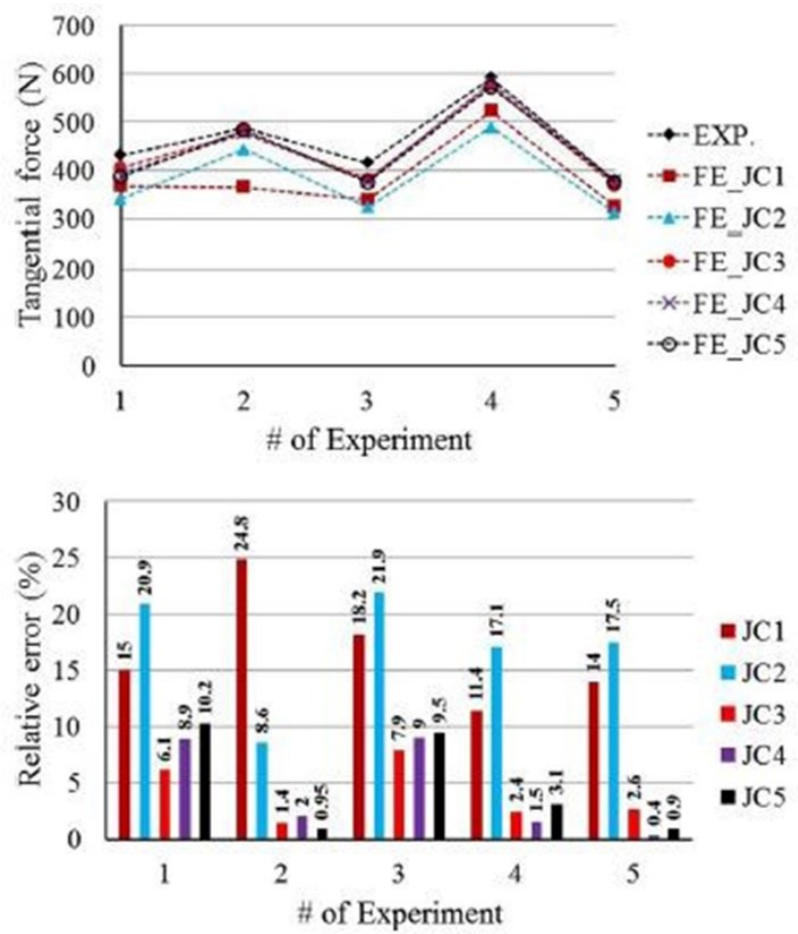

Fig. 7. Comparison between experimental (EXP) and predicted (FE) tangential forces [2].

As a conclusion of his paper, the analysis of the predicted cutting parameters shows that the material constants set obtained with 0 degrees rake angle gives an accurate prediction of chip thickness, cutting forces and tool-chip contact length.

Zhang [3] aims to investigate in his paper the mechanical properties of $7 \mathrm{~N} 01$ aluminium alloy under qui-static and dynamic condition. For this to be done, the author presents a modified J-C model for 7N01 aluminium alloy which was developed based on experimental observations. Also, the paper presents numerical simulations of the dynamic tensile tests, being performed using the finite element code ABAQUS, with the modified Johnson-Cook model (Fig.8).

As a conclusion of the paper, a modified Johnson-Cook constitutive equation is proposed by the author, whose coefficient of strain rate hardening parameter $\mathrm{C}$ is modified to a function related to strain rate. The results show that the modified model matched the experimental results better compared to the original Johnson-Cook model.

Shokry [4] presents in his paper a modified Johnson-Cook model for the flow behaviour of alloy $800 \mathrm{H}$ at intermediate strain rates and high temperatures. The author is using the Kalman filter technique in order to obtain the parameters that constitute the modified model and the original Johnson-Cook model. 


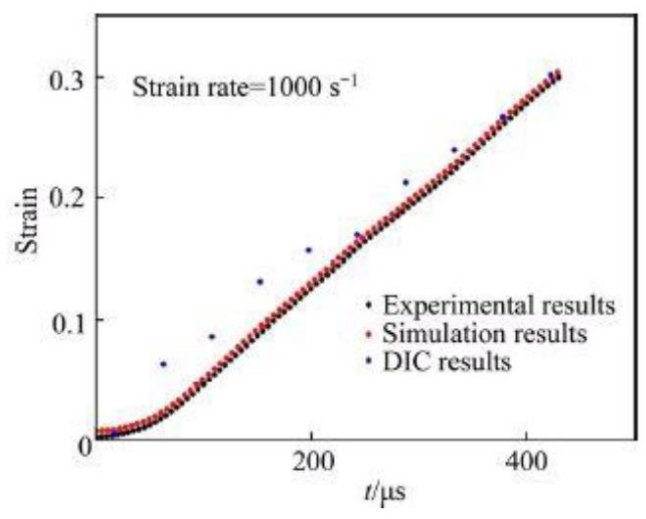

Fig. 8. Comparison between experimental results, simulation results and DIC (Digital Image Correlation) results in a true strain versus time curve [3].

The results show that the predicted stresses of the modified model fit the experimental data better than the predicted stresses got through the original Johnson-Cook model, as it can be seen in figure 9 .
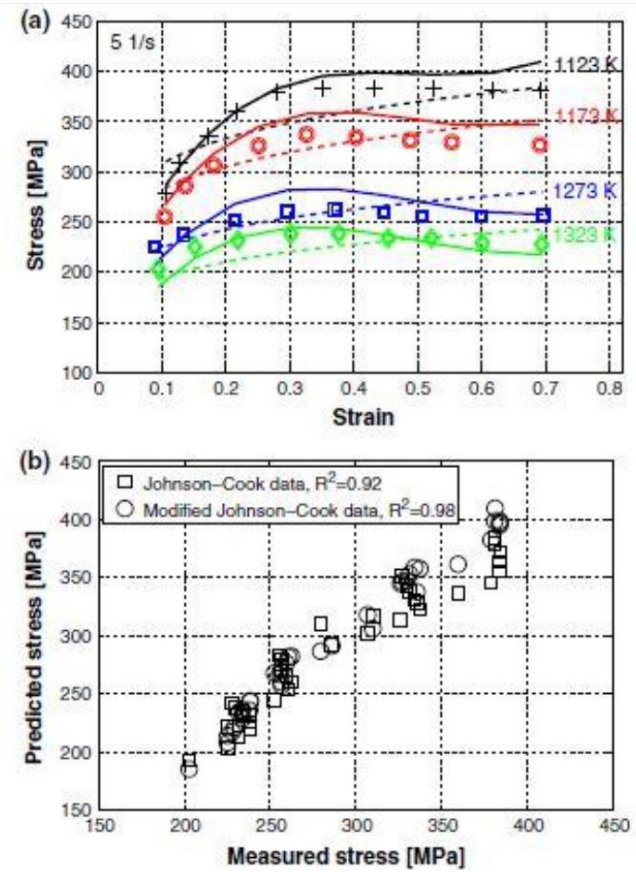

Fig. 9. Comparison between predicted stresses and measured stresses, using both original JohnsonCook model (marked with the dashed lines) and the modified Johnson-Cook model (marked with solid lines) at strain rate [4].

As a conclusion of his paper, the author aims that the modified model gives a more accurate prediction of the flow behaviour of alloy $800 \mathrm{H}$ than the original Johnson-Cook model, even for the measured stresses that were not included in the calculation processes of the parameters.

Rasaee [5] studied in his paper the hot compression behaviour of A12024. For this study the author uses the following ranges: 
- Temperatures between 573 and $723 \mathrm{~K}$,

- $\quad$ Strain rate between 0.001 and $0.6 \mathrm{~s}-1$.

The prediction of flow stresses was performed using constitutive equations based on the basic and modified Johnson-Cook model, and the accuracy of the proposed models was estimated by statistical error analysis method. As it can be seen in figure 10, flow stress got changed significantly, with changes in the strain rate and temperature (graphs based on the experimental results).
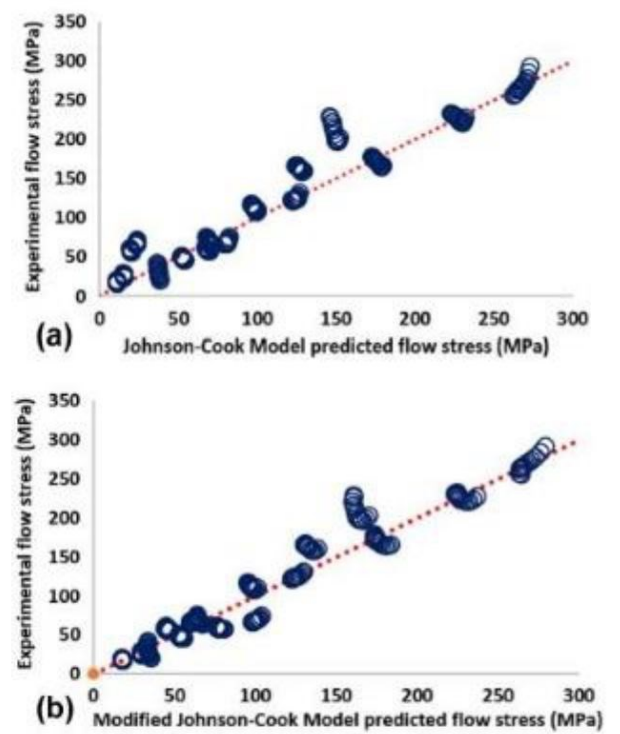

Fig. 10. Comparison between predicted flow stress and the experimental flow stress [5].

Also, as a conclusion of the paper, the author shows that during calculation, the constants in the modified model were improved, the effects of hardening and softening behaviour were included in addition, the correlated effects of the parameters was taken into account and all these leads to an significantly increase of the accuracy of the modified model.

Buzyurkin [6] presents in his study the analysis and verification of mathematical relations that describe deformation and fracture of the fan casing in a high-velocity impact with a fan blade simulator. Based on available experimental data, stress-strain curves were constructed for three titanium-based alloys (VT6, OT4, and OT4-0) under quasi-static loading. These alloys are used in fabrication of fan casings in aviation engines. The parameters of the Johnson-Cook model for the examined alloys were determined. Results of LS-DYNA simulations of blade simulator interaction with the fan casing are presented.

In his paper Huang [7] presents a multivariable identification method for J-C constitutive model. The author is using an algorithm called CGOA (cluster global optimization algorithm) in order to obtain a faster and accurate way for parameters determination of the virtual material and also for two real materials, namely 401 steel and 823 steel. The difference between this algorithm and the traditional manual method is that the second one obtains the parameters in sequence. The results show that the relative percentage error between the optimized and assumed parameters is no more than $4.02 \%$. This result illustrates the feasibility, accuracy and efficiency of using CGOA in the application of Johnson-Cook model parameters determination. 
Also in papers [8] and [9] there are presented cases where the Johnson-Cook model was used in order to analyse further aspects of the material deformation.

Due to the fact that the caulking operation is not studied at a high level, the conclusion is that a Johnson - Cook model is necessary to be implemented for this process.

\section{SWOT analyses for using Johnson-Cook model for caulking process}

Taking into consideration all the facts presented in the previous chapter and the lack of studies regarding the caulking operation the following step is to develop a SWOT analysis of the necessity of using Johnson-Cook model for the caulking:

- $\quad$ Strengths

$\circ \quad$ World-wide usage of Johnson-Cook model;

- Possibilities of comparison between different models;

- Approaching a different view for the caulking operation.

- Weaknesses

○ Lack of studies for the caulking operation;

- Small range of using Johnson-Cook model for this kind of deformation.

- Opportunities

○ Developing an analytical model for the entire domain of caulking operations;

- Threats

$\circ$ The emergence of other models to combat the principles of the JohnsonCook model.

Based on the SWOT analysis, it can be concluded that the usage of the Johnson-Cook model can bring significant improvements to the existing studies of the caulking operation.

\section{Conclusion}

The purpose of this paper is to investigate the major input data needed to perform an analysis with finite elements for the process of caulking.

The paper highlights the importance and the necessity of using the analytical JohnsonCook model for the study of caulking operation.

Several studies are presented in order to obtain a bigger image of all the test cases and domains where different materials (mainly alloys of aluminium and steel) and process parameters are being used in detailed analysis using Johnson-Cook model.

Taking all this information into consideration, a SWOT analysis was conducted with the output that the analytical model is able to bring significant improvements for an operation which is not studied at the level of importance at it is used, like the caulking process is.

The study is also a base for the following researches which will be developed, for the comparison between analytical and experimental studies.

\section{References}

1. L. Schwer, $6^{\text {th }}$ Eur LS-DYNA (2007)

2. M. Daoud \& J. F. Chatelain \& A. Bouzid, Int J Adv Manuf Technol, 81 (2015)

3. Y. Zhang, Y. Song, H. Xiang, W. Zhong-gang, J. Cent. South Univ, 24 (2017)

4. A. Shokry, JMEPEG, 26 (2017)

5. S. Rasaee, A. H. Mirzaei, Trans Indian Inst Met, (2019) 
6. A. E. Buzyurkin, I. L. Gladkyb and E. I. Kraus, Journal of Applied Mechanics and Technical Physics, (2015)

7. Z. Huang, L. Gao, Y. Wang, and F. Wang, JMEPEG, 25 (2016)

8. J. Ning,Y. Liang, The Int Journal of Adv Manufacturing Technology 97 (2018)

9. J. Cai, K. Wang, JMEPEG, 24 (2015) 